\title{
Effect of a 1-Year Controlled Lifestyle Intervention on Body Weight and Other Risk Markers (the Healthy Lifestyle Community Programme, Cohort 2)
}

\author{
Christian Koeder ${ }^{a, b}$ Ragna-Marie Kranz ${ }^{b}$ Corinna Anand ${ }^{b}$ Sarah Husain $^{b}$ \\ Dima Alzughayyar ${ }^{b}$ Nora Schoch ${ }^{b}$ Andreas Hahn ${ }^{a}$ Heike Englert $^{\text {b }}$

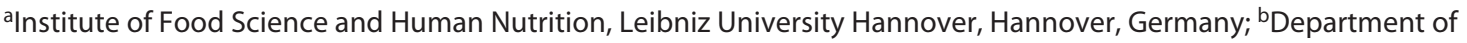 \\ Nutrition, University of Applied Sciences Münster, Münster, Germany
}

\section{Keywords}

Plant-based diet - Overweight · Lifestyle medicine ·

Preventive medicine $\cdot$ Cardiovascular disease

\begin{abstract}
Introduction: The prevalence of obesity is high and increasing worldwide. Obesity is generally associated with an increased risk of chronic disease and mortality. The objective of the study was to test the effect of a lifestyle intervention on body weight and other chronic disease risk markers. Methods: A non-randomized controlled trial was conducted, including mostly middle-aged and elderly participants recruited from the general population in rural northwest Germany (intervention: $n=114$; control: $n=87$ ). The intervention consisted of a 1-year lifestyle programme, focussing on four key areas: a largely plant-based diet (strongest emphasis), physical activity, stress management, and community support. Parameters were assessed at baseline, 10 weeks, 6 months, and 1 year. The control group received no intervention. Results: Compared to the control, in the intervention group, significantly lower 1-year trajectories were observed for body weight, body mass index (BMI), waist circumference (WC), total cholesterol, calculated LDL choles-
\end{abstract}

terol, non-HDL cholesterol, remnant cholesterol (REM-C), glucose, $\mathrm{HbA1C}$, and resting heart rate (RHR). However, between-group differences at 1 year were small for glucose, $\mathrm{HbA1c}$, and cholesterol (apart from REM-C). No significant between-group differences were found for 1-year trajectories of measured LDL cholesterol, HDL cholesterol, triglycerides, insulin, blood pressure, and pulse pressure. Conclusion: The intervention successfully reduced body weight, BMI, WC, REM-C, and RHR. However, at 1 year, effectiveness of the intervention regarding other risk markers was either very modest or could not be shown.

(c) 2021 The Author(s).

Published by S. Karger AG, Basel

\section{Introduction}

Worldwide, the prevalence of obesity has been increasing for several decades, and the situation has been described as a pandemic $[1,2]$. In Germany, as in many other countries, more than $20 \%$ of adults have obesity [1]. Obesity is generally associated with an increased risk of chronic diseases and, in the general population, increased cardiovascular disease (CVD) and all-cause mortality [3, 4]. Complex reasons for obesity have been proposed, and karger@karger.com www.karger.com/ofa

Karger $\stackrel{\text { ' }}{5}$

BOPEN ACCESS
(C) 2021 The Author(s)

Published by S. Karger AG, Basel

This is an Open Access article licensed under the Creative Commons Attribution-NonCommercial-4.0 International License (CC BY-NC) (http://www.karger.com/Services/OpenAccessLicense), applicable to the online version of the article only. Usage and distribution for commercial purposes requires written permission.
Correspondence to:

Christian Koeder, koeder@fh-muenster.de 
there is a range of factors to consider in terms of the aetiology of obesity $[1,2,5,6]$. While such environmental, psychological, and metabolic factors can constitute barriers to putting a healthy lifestyle into practice $[1,7]$, the actual implementation of healthy lifestyle patterns could drastically lower the public health and individual burdens of obesity [8]. Lifestyle intervention programmes can serve as effective tools for addressing such barriers [9].

While diet and physical activity both are important for weight loss, diet can be considered the most critical factor $[1,10]$. An effective lifestyle approach to address obesity may be the recommendation to follow a plant-based diet, i.e., a dietary pattern that is centred around health-promoting foods of plant origin [11-13]. A traditional Mediterranean diet is one example of such a plant-based dietary pattern which is focused on high intakes of fruit, vegetables, whole grains, legumes, nuts, seeds, and healthy oils [14]. Unless alcohol or oil intake is excessive, such a dietary pattern is high in fibre, has a low caloric density (compared to a typical "Western" dietary pattern), and promotes a healthy body weight [15]. In contrast, dietary interventions that focus on caloric restriction [16-18] are frequently unsuccessful in the long term [1] and may leave the individual constantly hungry [19], which may then result in a relapse to previous dietary behaviours [20]. Furthermore, dietary weight loss strategies with a simplistic focus on low-fat [21,22] and low-sugar products [1] may be flawed and may even have adverse effects [21]. Certain plant-based foods such as nuts/avocados (high-fat) and fruits (high-sugar), which are generally regarded as healthy, would be excluded, if solely macronutrient composition is considered, instead of considering both macronutrient content as well as overall food quality.

Lifestyle interventions can improve health behaviour, including diet [23], physical activity [24,25], smoker status [26], and possibly sedentary behaviour [27, 28]. Lifestyle interventions have been shown to improve disease risk markers, including body weight, body mass index (BMI), blood pressure, fasting blood glucose, and $\mathrm{HbAlc}$ $[23,29,30]$.

While education-based lifestyle interventions can be effective [25], it is likely that effectiveness can be increased if interventions also incorporate strategies for offering practical guidance $[31,32]$ and for increasing participants' motivation [33]. The use of nudging, goal setting, and progress monitoring, for example, can motivate participants and increase adherence to recommendations $[34,35]$. Furthermore, social support can facilitate successful lifestyle modification [10]. In contrast, body weight stigma is harmful and may promote the psycho- logical issues that are frequently related to unhealthy eating practises and obesity $[36,37]$.

Frequently, lifestyle interventions have been able to demonstrate only short-term successes [38]. In addition, many dietary interventions have only assessed short-term outcomes ( 3-4 months), and many have exclusively focused on certain dietary components (fruit, vegetables, fat, etc.) rather than whole dietary patterns [32]. Furthermore, many controlled studies lack a no-intervention control group [32], and while high-risk individuals are often recruited for lifestyle programmes, community-based interventions are more inclusive, can reach individuals outside of conventional healthcare settings, can provide expertise not easily accessible otherwise to citizens, and can have a snowball effect on the wider community [39].

Against this background, we hypothesized that a lifestyle intervention would effectively improve body weight and other chronic disease risk markers in a communitybased setting, i.e., in a heterogeneous sample of participants from the general population (most of whom were clinically healthy). The objective of the study was to test the effectiveness of the intervention in the context of community health promotion.

\section{Materials and Methods}

\section{Study Design}

We conducted a non-randomized, controlled intervention trial between April 2018 and October 2020. Measurements were taken at baseline, 10 weeks, 6 months, and 1 year. Planned measurements for 18 months and 2 years could not be included due to the COVID-19 pandemic (18-month time point: uneven time delays; intervention: September 2019, 17 months; control: June 2020, 20 months; results including the 18-month time point are shown in online supplementary Table 1 [for all online suppl. material, see www.karger.com/doi/10.1159/000521164]; 2-year time point: intervention: July 2020; no assessment in the control group). Participants were recruited from the general population in rural northwest Germany (intervention group: February 2018; control group: September 2018; as described previously [40]).

The intervention consisted of a healthy lifestyle programme, whereas the control group received no intervention. Participants were not blinded. Staff performing laboratory assessments were unaware of group allocation. Participants were not randomized because the intervention and control groups were recruited in two separate municipalities. The reason for this was that participants of the control group were meant to be unaware of the contents of the intervention. As we were unable to recruit and start both study arms at the same time, the control group study arm started and finished 6 months later (start: October 2018) than the intervention group (start: April 2018), with equivalent follow-up intervals in both groups. The study was registered in the German Clinical Trials Register (DRKS; reference: DRKS00018775; www.drks.de). 
Fig. 1. Flow chart of participants through the study.

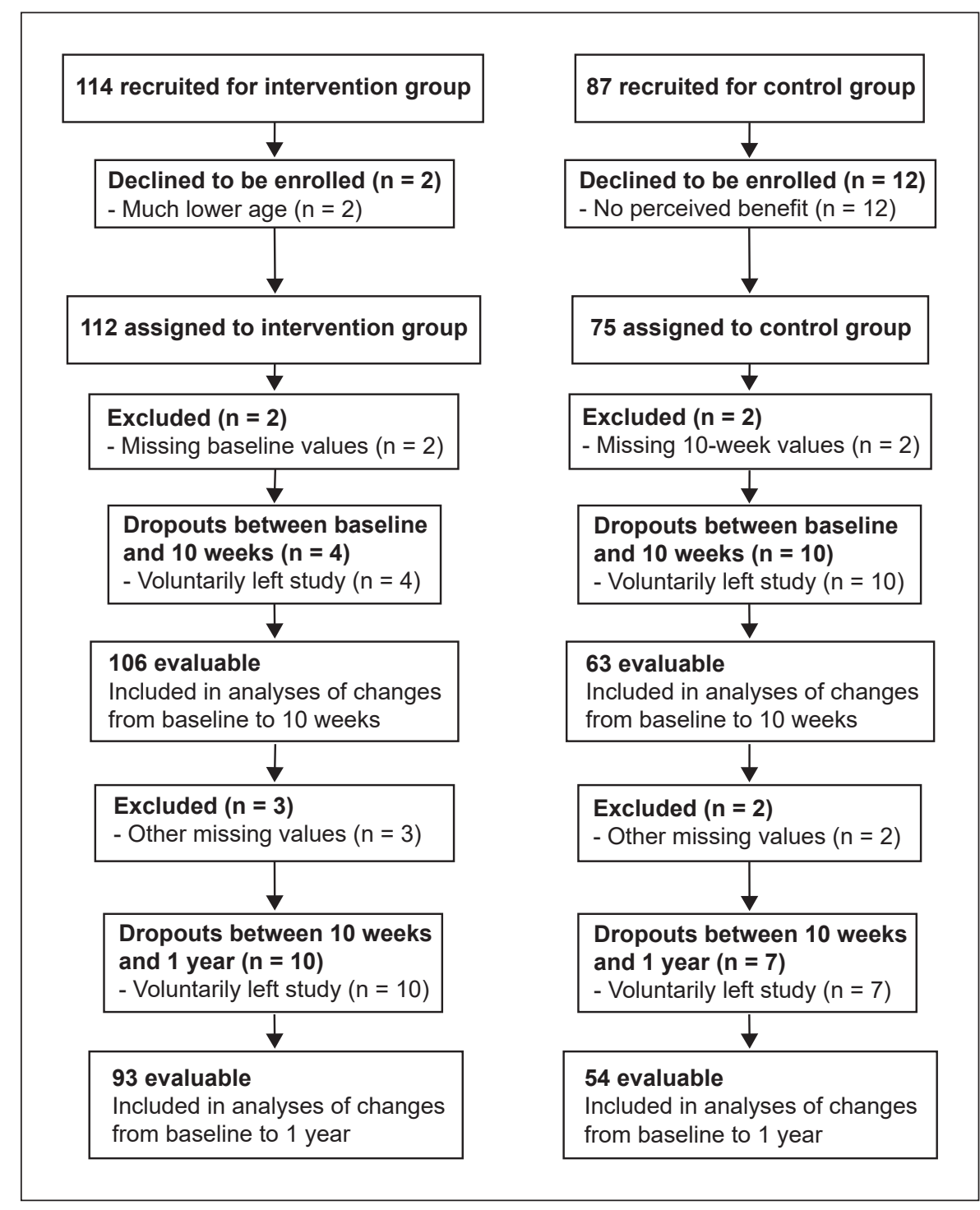

\section{Participants}

Participants were mostly middle-aged and elderly. The only inclusion criteria were the physical and mental ability to take part in the study (self-reported) and to be $\geq 18$ years old. For the intervention, a total of 114 and for the control group, a total of 87 participants were recruited (shown in Fig. 1).

\section{Lifestyle Programme}

The lifestyle intervention (Healthy Lifestyle Community, cohort 2) consisted of an intensive phase (first 10 weeks) and a less intensive phase (remainder of the study). The intensive phase consisted of 14 seminars (each with $\sim 70-100$ participants and 3-4 health professionals) and 8 workshops (duration: $\sim 2 \mathrm{~h}$ each, as described previously [40]). During the seminar breaks ( $15 \mathrm{~min})$, healthy snacks were provided (first by the research team, then increasingly by the participants), for example, whole-grain bread with plant-based spreads, salads, fruit, nuts, etc. Seminar topics included causes and development of lifestyle-related diseases, risk factors, lifestyle choices, and behaviour change (based on Prochaska's model of change $[26,41]$ and transtheoretical model $[42,43])$. The seminars also included practical interactive sessions $(\sim 10-30$ min; for example, cooking, exercise, meditation, and group work). Evidence-based content was presented using tangible examples and everyday language, while including vivid study materials (printed; basic literacy level) and employing the concepts of nudging [34] and gamification [44]. In one seminar, issues related to sex-specific diseases (such as postmenopausal weight gain and prostate cancer) were discussed in separate groups for women and men to provide a safe space for questions [45]. Seminars and workshops were conducted by our research group. Lifestyle choices were addressed in four key areas: diet, physical activity, stress management, and community support [13]. The strongest emphasis was on dietary change, with the recommendation of a healthy, plant-based diet $[11,16]$. Specifically, recommendations were to consume more fruit, vegetables, whole grains, legumes (including soya foods), nuts, seeds, and healthy oils (such as cold-pressed ol- 
ive or rapeseed oil) and to decrease the intake of meat (including red meat and poultry), butter, full-fat dairy, eggs, salt, and added sugars as well as to avoid alcohol excess [12]. There was no specific target in terms of the percentage of calories from different macronutrients. Non-dietary lifestyle recommendations included to be physically active for at least $30 \mathrm{~min}$ per day, to establish relaxation routines, and to spend more time with others (specific recommendations were not given in these areas).

Participants received a healthy lifestyle handbook, a recipe booklet, and a laminated information sheet with an overview of the lifestyle recommendations. They also took part in two one-on-one coaching sessions ( 15 min each; at baseline and 10 weeks).

\section{Assessment of Parameters}

Measurements and blood sampling were all performed in the morning and in the fasted state. All analyses of blood samples were conducted at the University Hospital of Münster (measurement protocols are listed in online suppl. Table 2). Semi-quantitative 3-day protocols were used to assess dietary intake: these included tally sheets for individual foods (with portion sizes), categorized by food groups (cereals and cereal products; vegetables and mushrooms; legumes and legume products; fruit; dairy; meat and fish; potatoes, side dishes, and sauces; cakes, sweets, and snacks; readymade meals; nuts and seeds; fats and oils; drinks; salt and sugar; and other foods/meals). The food protocols also contained several illustrated portion sizes (using examples such as "a handful" and "the size of your palm") and included two weekdays and one weekend day. The food protocols were tailored to the study population but were not validated. Adherence to dietary recommendations was assessed with the plant-based diet index (PDI), healthful PDI (hPDI), and unhealthful PDI (uPDI) [46] (due to the nature of our data, instead of reverse scores based on quintiles, as described by Satija et al. [46], we used positive and negative scores based on food portions; for example, for the calculation of the PDI score, all animal-source food portions were subtracted from all plant-based food portions). These indexes are based on 18 food groups. Higher scores for PDI and hPDI and lower scores for uPDI are considered favourable. Dietary recommendations in our study were partly based on the hPDI. Socio-demographic parameters and physical activity (in categories) were assessed using questionnaires.

\section{Study Hypotheses}

The primary hypothesis of the study was that in a heterogeneous sample from the general population (living lab approach), the intervention would lead to significantly reduced body weight, within the intervention group and compared to control, both at 10 weeks and at 1 year. Similarly, secondary hypotheses were that the intervention would significantly reduce (compared to control) BMI, waist circumference (WC), total cholesterol (TC), measured LDL cholesterol (LDL-C), calculated LDL-C, triglycerides (TAG), glucose, $\mathrm{HbA1c}$, insulin, systolic and diastolic blood pressures (BPs), and resting heart rate (RHR). HDL cholesterol (HDL-C) was assessed exploratively. The analyses of non-HDL cholesterol (non-HDL-C), remnant cholesterol (REM-C), and pulse pressure $(\mathrm{PP})$ were non-prespecified.

\section{Statistical Analyses}

A sample size calculation was performed based on change in body weight, the primary outcome parameter of the study. This calculation was based on data from a pilot study with a prototype version of the lifestyle programme (as described previously [40]). Assuming a dropout rate of at least $10 \%$, a minimum sample size of 93 participants (intervention: 62; control: 31 ) was indicated to reach a global power of 0.8 and a global significance level of 0.05 .

For between-group comparisons of baseline characteristics, Fisher's exact test was used for categorical variables. The independent $t$ test was used for normally distributed variables, and the Mann-Whitney U test for non-normally distributed continuous variables. The Shapiro-Wilk test was used to assess data for nonnormality, and $p<0.05$ was defined as describing a non-normal distribution. To evaluate within-group changes, a paired $t$ test was used for normally distributed data, and the Wilcoxon test was used for non-normally distributed data. All tests were two-sided.

For the analyses of changes from baseline to 10 weeks, betweengroup differences were assessed with a one-way analysis of covariance (ANCOVA). For the analyses of changes from baseline to 1 year, a one-way repeated-measures ANCOVA was used, using potential confounders as covariates.

Bivariate correlations were assessed with Spearman's rho correlations (two-sided). Analyses were based on unimputed data (complete case analysis [CCA]). In sensitivity analyses, imputed data (last observation carried forward [LOCF]) were used. Blinding was not feasible for statistical analysis. The analysis strategy was intention to treat (participants were included in analyses irrespective of compliance). Statistical significance was consistently set at the 0.05 level. All analyses were conducted using IBM SPSS Statistics (version 25.0; Armonk, New York, NY, USA).

\section{Results}

\section{Baseline Characteristics}

The flow of participants through the study is shown in Figure 1. These participants were included in the analyses (CCA).

Compared to the control, at baseline, the intervention group had a higher mean age $(p=0.003)$, higher educational level $(p=0.002)$, and higher mean REM-C $(p=0.015)$ (Table 1$)$. In addition, the intervention group had a higher (more favourable) baseline hPDI score compared to the control $(p<0.001)$. Baseline alcohol intake frequency and physical activity levels were not significantly different between groups $(p>0.05)$. In terms of other characteristics, both groups were similar (Table 1).

For both groups combined, those with missing values (non-evaluable participants) were more likely to have obesity $(p=0.046)$, to be male $(p=0.035)$, or to be smokers $(p=0.010)$. They had a higher body weight $(p=0.009)$ and WC $(p=0.028)$, higher REM-C $(p=0.020)$, TAG ( $p$ $=0.018)$, and insulin $(p=0.031)$ as well as lower HDL-C $(p=0.001)$. In the intervention group, those with missing values were more likely to be smokers $(p=0.009)$ and had lower HDL-C $(p=0.013)$. In the control group, those with 
Table 1. Baseline characteristics of evaluable participants (CCA)

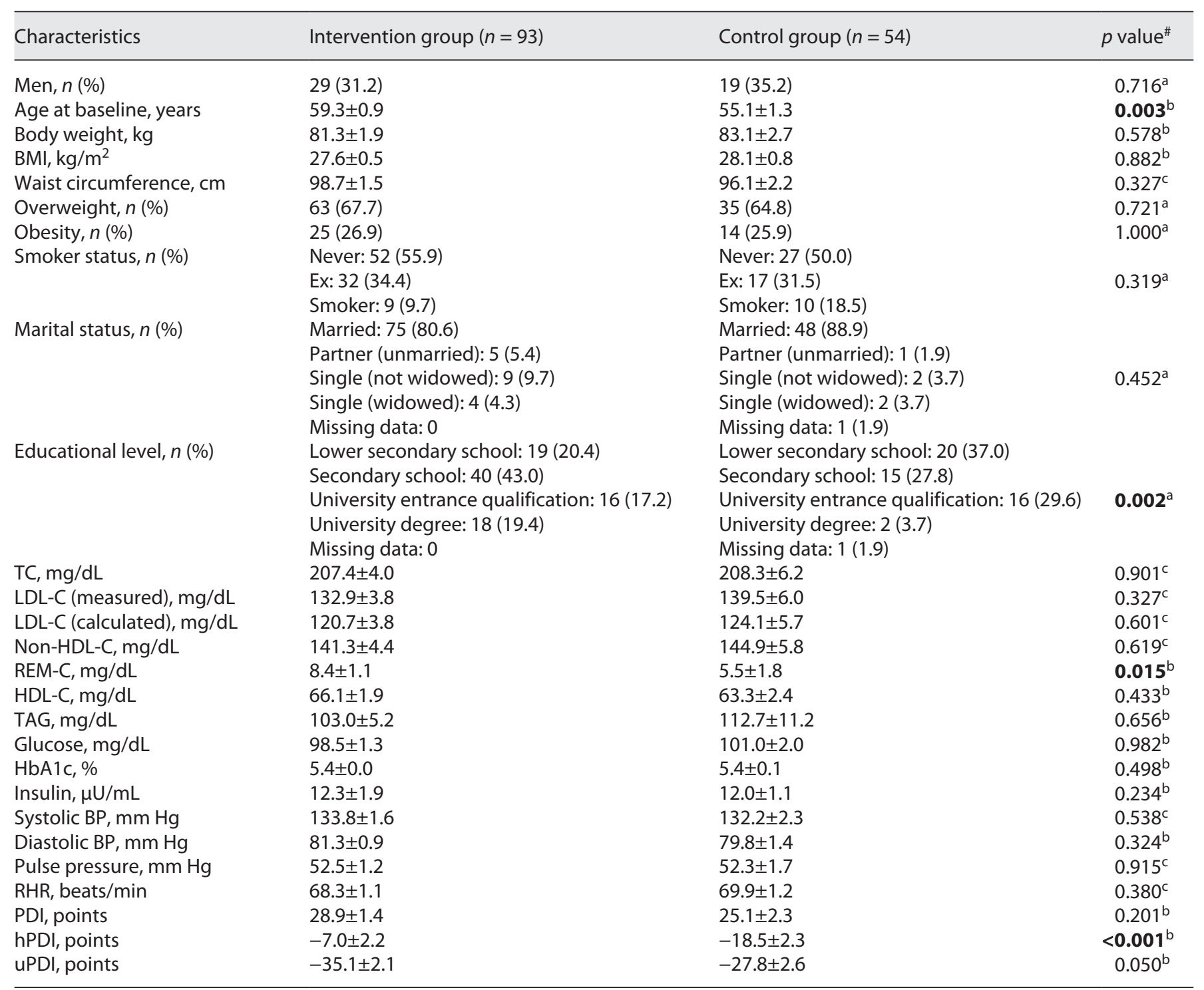

Values are means $\pm \mathrm{SEM}$, except for qualitative variables, expressed as $n$ (\%). TC, measured LDL-C, non-HDL-C, REM-C, HDL-C, TAG, glucose, HbA1C, and insulin: $n=92$ (intervention), $n=53$ (control); calculated LDL-C: $n=92$ (intervention), $n=52$ (control); systolic/diastolic BP, PP, and RHR: $n=52$ (control); PDI, hPDI, and uPDI: $n=91$ (intervention), $n=53$ (control). SEM, standard error of the mean. ${ }^{\#} p$ value for between-group comparisons by ${ }^{\mathrm{a}}$ Fisher's exact test (two-sided) and the ${ }^{\mathrm{b}}$ Mann-Whitney $\mathrm{U}$ test (two-sided) and ${ }^{\mathrm{C}}$ independent $t$ test (twosided).

missing values were more likely to be male $(p=0.027)$, had a higher body weight $(p=0.008)$ and WC $(p=0.017)$, higher REM-C $(p=0.014)$, and lower HDL-C $(p=0.048)$.

\section{Seminar Attendance}

Seminar attendance during the 10-week intensive phase of the intervention was relatively high: 75 out of 106 participants evaluable for 10-week analyses (70.8\%) and
69 out of 93 participants evaluable for 1-year analyses (74.2\%) attended $\geq 11$ (out of 14 ) seminars.

\section{Changes in Body Weight and Risk Markers from}

Baseline to 10 Weeks (Intensive Phase; CCA)

Compared to the control, from baseline to 10 weeks in the intervention group, statistically significant reductions were observed for body weight, BMI, WC, TC, measured 
Table 2. Baseline and follow-up measurements in evaluable participants (IN: $n=93$; CON: $n=54$ )

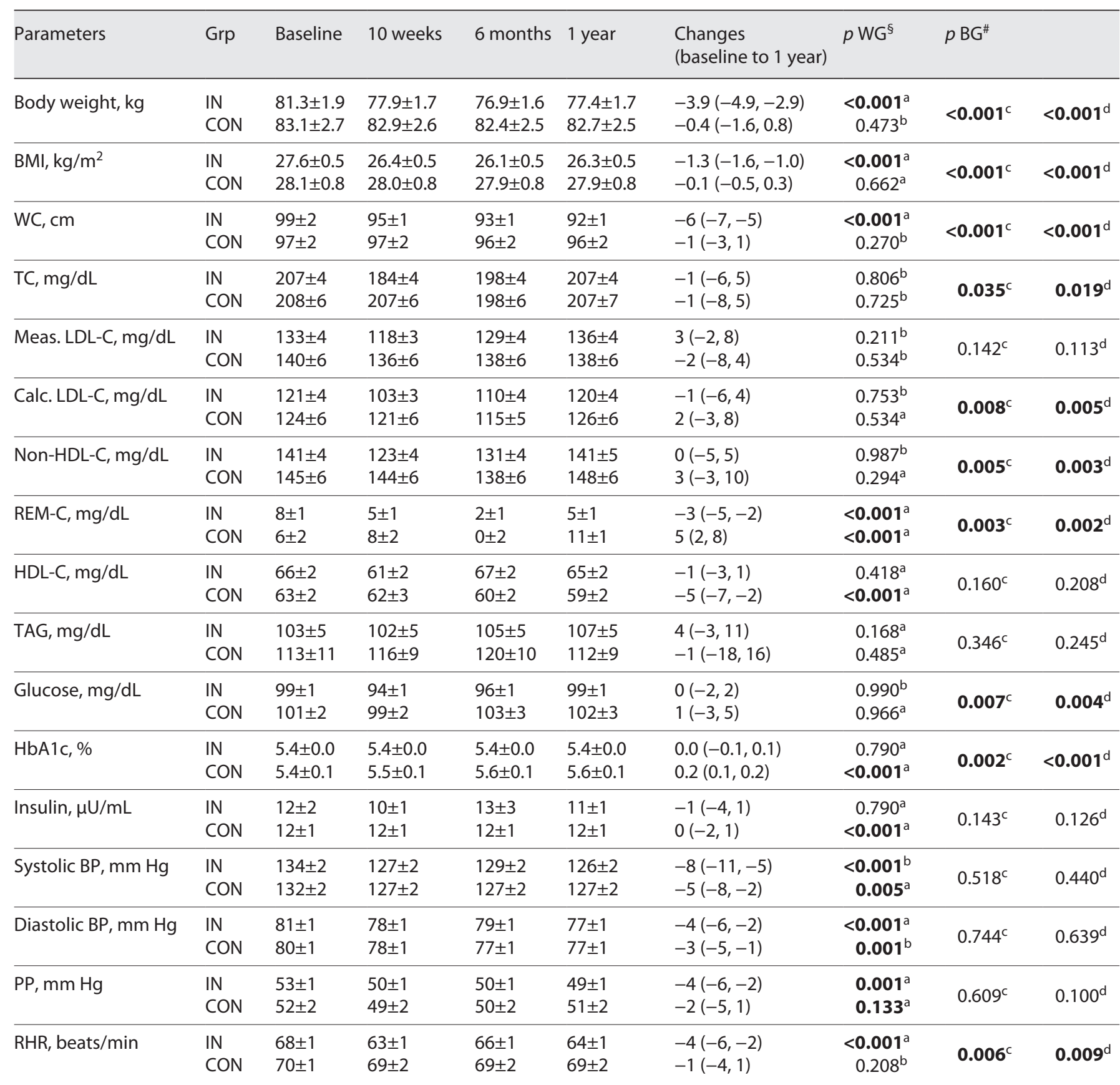

Values are means $\pm \mathrm{SEM}$, except for qualitative variables which are expressed as $n$ (\%). Changes are expressed as means and $95 \% \mathrm{Cl}$; WC: $n=53$ (CON); TC, measured LDL-C, non-HDL-C, REM-C, HDL-C, TAG, glucose, and insulin: $n=92$ (IN), $n=53$ (CON); calculated LDL-C: $n$ $=92(\mathrm{IN}), n=52$ (CON); HbA1c: $n=91$ (IN), $n=53$ (CON); systolic/diastolic BP, PP, and RHR: $n=52$ (CON). SEM, standard error of the mean; $\mathrm{Cl}$, confidence interval; $p$ WG, $p$ values for within-group changes from baseline to 1 year; $p$ BG, $p$ values for between-group differences in 1-year trajectories; IN, intervention; CON, control; Grp, group; Meas. LDL-C, measured LDL, cholesterol; Calc. LDL-C, calculated LDL-C. ${ }^{\S} p$ value for within-group comparisons by the ${ }^{a}$ Wilcoxon test (two-sided) and ${ }^{b}$ paired $t$ test (two-sided). ${ }^{\#} p$ value for between-group comparisons by ${ }^{C}$ repeated measures ANCOVA, adjusted for the baseline and ${ }^{d}$ repeated measures ANCOVA, adjusted for baseline, age, and sex.

Healthy Lifestyle Intervention to Address Overweight and Other Risk Markers 
Fig. 2. One-year trajectories of hPDI score changes from baseline (portions/day). Values are means and 95\% CIs of hPDI score changes (food portions/day; adjusted for baseline); ${ }^{*} p$ value for differences from baseline: $p \leq 0.001$. CI, confidence interval.

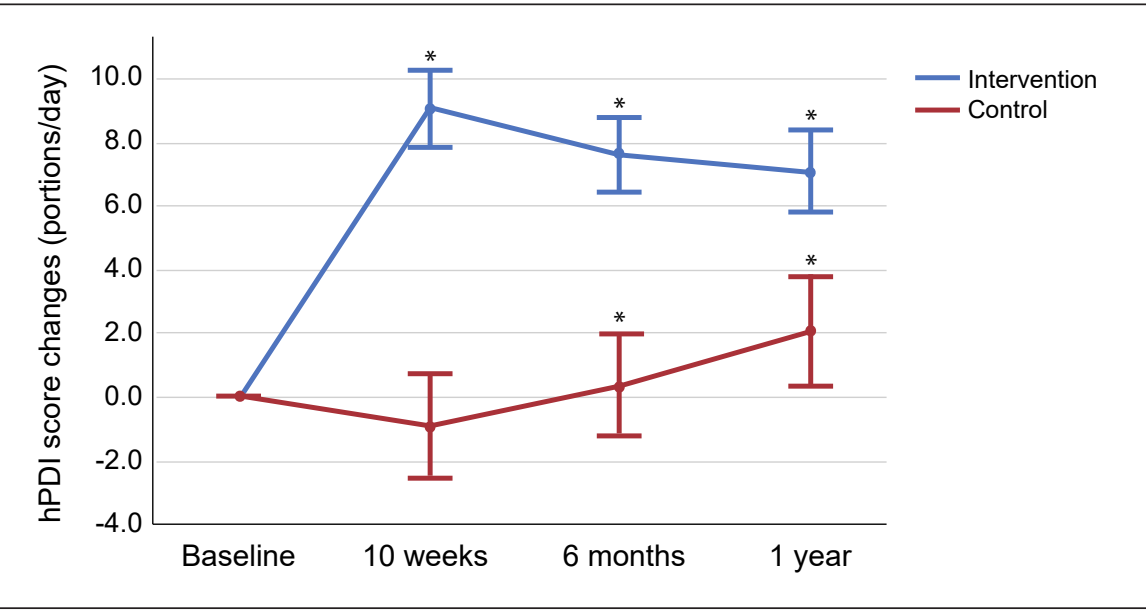

and calculated LDL-C, non-HDL-C, REM-C, HDL-C, glucose, insulin, and RHR (all: $p \leq 0.025$; adjusted for baseline values; online suppl. Table 3 ). No significant between-group differences were observed for TAG, $\mathrm{HbA1c}$, systolic or diastolic BP, or PP (adjusted for baseline values; online suppl. Table 3). In sensitivity analyses with imputed data (LOCF), these results were confirmed, except for TAG, which was significantly reduced in the intervention group compared to the control ( $p=0.044$; adjusted for baseline values, age, and sex).

\section{Changes in Body Weight and Risk Markers from}

Baseline to 1 Year (CCA)

Compared to the control, in the intervention group, significantly lower 1-year trajectories (including all time points) were observed for body weight, BMI, WC, TC, calculated LDL-C, non-HDL-C, REM-C, glucose, HbA1c, and RHR (Table 2). No significant between-group differences were observed for the 1-year trajectories of measured LDL-C, HDL-C, TAG, insulin, systolic or diastolic $\mathrm{BP}$, or PP (adjusted for baseline values; Table 2). In sensitivity analyses with imputed data (LOCF), these results were confirmed, except for TC and glucose, for which there were no significant between-group differences.

Adjustments for age and sex confirmed the results. Additional adjustments for smoker status, educational level, marital status, BMI, REM-C, and changes in alcohol intake confirmed these results.

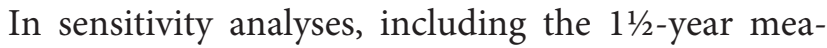
surement time points, these results were largely confirmed. However, for glucose and RHR, there were no significant between-group differences (adjusted for baseline values; online suppl. Table 1).
Dietary Changes from Baseline to 10 Weeks (Intensive Phase)

In the intervention group, dietary scores significantly improved from baseline to 10 weeks and significantly more so than in the control: in the intervention group, PDI increased by 11 points ( $~ 4$ portions/day) and hPDI increased by 23 points ( $\sim 8$ portions/day), while uPDI decreased by 11 points ( $\sim 4$ portions/day; unadjusted; all: $p$ $<0.001)$.

\section{Dietary Changes from Baseline to 1 Year}

At 1 year, in the intervention group, PDI and hPDI were still increased (compared to baseline) by 9 points ( $\sim 3$ portions/day) and 19 points ( 6 portions/day), respectively, while uPDI was still decreased by 11 points ( $\sim 4$ portions/day; unadjusted; all: $p<0.001$ ). In Figure 2, changes in the hPDI score (adjusted for baseline) in both groups are shown.

\section{Bivariate Correlations between Diet Score Changes and Risk Marker Changes (Baseline to 10 Weeks)}

In bivariate correlations (including participants of both groups), PDI change inversely correlated with changes in body weight, WC, TC, LDL-C (measured and calculated), non-HDL-C, REM-C, and HDL-C (all: $p \leq$ 0.042; online suppl. Table 4 ). Inverse correlations were observed between hPDI change and changes in body weight, BMI, WC, TC, LDL-C (measured and calculated), non-HDL-C, REM-C, HDL-C, insulin, and RHR (all: $p \leq$ 0.046; online suppl. Table 4). Positive correlations were observed between uPDI change and changes in body weight, WC, glucose, and RHR (all: $p \leq 0.031$; online suppl. Table 4). 
Bivariate Correlations between Diet Score Changes and Risk Marker Changes (Baseline to 1 Year)

Similar correlations were observed regarding changes from baseline to 1 year (expressed as the difference between baseline and the mean of the three follow-up time points): $\mathrm{PDI}$ change inversely correlated with changes in body weight, BMI, WC, TC, non-HDL-C, REM-C, glucose, HbAlc, and insulin (all: $p \leq 0.026$ ). Inverse correlations were also observed between hPDI change and changes in body weight, BMI, WC, TC, non-HDL-C, LDL-C (measured and calculated), HbA1c, and RHR (all: $p \leq 0.027$ ). Positive correlations were observed between uPDI change and changes in body weight, BMI, WC, HbAlc, and RHR (all: $p \leq 0.029$ ).

Bivariate Correlations between Diet Score Changes and Risk Marker Changes (10 Weeks to 1 Year)

For changes from 10 weeks to 1 year, inverse correlations were observed between hPDI change and changes in TC, HDL-C, and RHR (all: $p \leq 0.033$ ). No statistically significant correlations were observed between changes in PDI or uPDI and changes in any of the assessed risk markers.

\section{Bivariate Correlations between Diet Score Changes} and Risk Marker Changes (Food Group Level)

When diet score changes at the food group level were correlated with corresponding risk marker changes, the above associations (at the PDI/hPDI/uPDI level) were largely confirmed.

\section{Physical Activity Changes (Baseline to 10 Weeks)}

From baseline to 10 weeks, significant increases in intense physical activity (minutes/week; $p=0.009$; sessions/ week; $p<0.001$ ), moderate physical activity (minutes/ week; $p=0.030$ ), and gentle physical activity (minutes/ week; $p=0.042$ ) were observed in the intervention group $(n=105)$ compared to the control $(n=60)$ (adjusted for baseline values, age, and sex).

\section{Physical Activity Changes (Baseline to 1 Year)}

The 1-year trajectory (including all time points) of intense physical activity (minutes/week) was still significantly higher in the intervention group $(n=90)$ compared to the control $(n=50 ; p=0.022$; adjusted for baseline values, age, and sex). However, this difference was due to a higher score in the intervention group at 10 weeks and 6 months. This higher score was not maintained at 1 year: in the intervention group, the difference in intense physical activity (minutes/week) between base-

Healthy Lifestyle Intervention to Address

Overweight and Other Risk Markers line and 1 year was not significant anymore $(p=0.916)$. No significant between-group differences were observed for the 1-year trajectories of moderate physical activity ( $p$ $=0.239)$, gentle physical activity $(p=0.110)$, or intense physical activity (sessions/week; $p=0.053$; adjusted for baseline values, age, and sex).

\section{Discussion}

The present study aimed at clarifying the effect of a multimodal 1-year lifestyle intervention on body weight and weight-related risk parameters in middle-aged and older subjects in a community-based setting. Adherence to dietary recommendations was largely maintained at 1 year. In the intervention group, significant reductions in body weight, BMI, WC, REM-C, and RHR were observed at 10 weeks, and these favourable changes were maintained at 1 year. The Unites States Preventive Services Task Force (USPSTF) recommendations (2018) state that effective, intensive, behaviour-based weight loss interventions are typically designed to help adults with obesity achieve a weight loss of $\geq 5 \%$ (through changes in diet and physical activity) [47]. Furthermore, the USPSTF recommendations state that after 1 year, a weight loss of $2-3 \mathrm{~kg}$ (compared to the control) is typically observed in such interventions (with an absolute weight loss ranging from 1 to $9 \mathrm{~kg}$ in the intervention groups) [47]. The European Practical and Patient-Centred Guidelines for Adult Obesity Management in Primary Care (2019) suggest that a weight loss of $5-10 \%$ of initial body weight is a realistic target as well as a measure of successful weight loss [48]. Participants with obesity in our intervention group $(n=24)$ reduced their body weight (compared to baseline) by approximately $4 \mathrm{~kg}(4 \%), 7 \mathrm{~kg}(7 \%)$, and $5 \mathrm{~kg}$ (5\%) within 10 weeks, 6 months, and 1 year, respectively (data not presented). This is in line with recommendations of $5-10 \%$ weight loss as a goal that can likely be maintained in the long term $[18,47,48]$.

While 1-year trajectories of TC, calculated LDL-C, non-HDL-C, and glucose were also significantly lower in the intervention group, these differences were due to strong improvements from baseline to 10 weeks, and at 1 year, these improvements were no longer clinically meaningful. One important factor contributing to this reversal of previously achieved improvements was likely diet. While in the intervention group hPDI (the most relevant of the three diet scores assessed [46]) was still clearly increased at 1 year compared to baseline, we observed a significant decrease in hPDI from 10 weeks to 1 year 
(i.e., after the end of the intensive phase; $p=0.014 ; n=$ 89; data not presented). Another relevant factor may have been that the significantly improved moderate and intense physical activity levels in the intervention group were maintained until 6 months (data not presented) but were not maintained at 1 year. This influence was likely small as in the intervention group, TC and LDL-C were seen to increase in the 10 weeks to 6 months timeframe. In addition, the influence of exercise on TC and LDL-C appears to be modest [49]. Dietary changes in the intervention group may also have been too moderate to achieve long-term cholesterol-lowering effects [50]. The results might also have been modestly influenced by a partial adaptation of endogenous cholesterol synthesis to dietary changes [51], seasonal changes [51, 52], and the relative imprecision of standard laboratory assessment methods [53]. It should be noted that seasonal changes may have influenced dietary intake: in the intervention group, hPDI greatly improved from spring to summer and decreased from summer to autumn and autumn to spring, while in the control group, hPDI slightly decreased from autumn to winter and increased from winter to spring and spring to autumn (Fig. 2). However, sensitivity analyses including the 11/2-year measurement time points largely confirmed the results (online suppl. Table 1).

It is also noteworthy that baseline HDL-C values were comparatively high in both groups $[54,55]$. At 1 year, the intervention group demonstrated a significant reduction in REM-C by about $3 \mathrm{mg} / \mathrm{dL}$ (a 38\% reduction from baseline), which is a modest but potentially clinically relevant improvement [56-59]. REM-C is a strong independent risk factor for CVD events and CVD mortality [56, 57]. In our study, an increased PDI score was associated with a decrease in REM-C. A healthy, largely plant-based diet (traditional Mediterranean or low-glycaemic) has been shown to be associated with lower REM-C [60].

The RHR was significantly lowered by about 4 beats/ $\mathrm{min}$ in the intervention group (compared to a decrease of 1 beat/min in the control group; Table 2), which is a clinically relevant effect [61]. The RHR is a risk marker for heart failure, stroke, cancer, and all-cause mortality [61, 62], and diet may be an underestimated factor for the RHR [63]. In our study, an increase in hPDI and a decrease in uPDI were associated with a decrease in the RHR. Regular aerobic exercise [63], consuming longchain omega-3 fatty acids [63], relaxation, giving up smoking [61], and substantial weight loss [64] are associated with a decrease in the RHR. In our study, we did not observe significant correlations between changes (base- line to 1 year) in body weight or physical activity and changes in the RHR (data not presented).

\section{Strengths and Limitations}

A strength of our study was the assessment of a large variety of parameters, multiple measurement time points, and a no-intervention control group. However, assessing multiple parameters also increases the likelihood of significant findings. Another strength is that our statistical analyses (ANCOVA) could adjust for various potential confounders. A limitation of our study was that the control group started with a delay of 6 months (same followup duration), compared to the intervention group. Seasonal variations may have influenced the results $[51,52]$. However, consistent seasonal trends in risk markers were not observed in either group (data not presented). A further limitation was that participants were not randomized (as described previously [40]). While both groups were comparable at baseline and we adjusted for potential confounders, some bias may have remained. While the dietary assessment method (3-day dietary record) provided relatively detailed data and avoided recall bias, it reflects only short-term food intake, and food intake may have been underreported, misreported, or adapted by the participants. In addition, our questionnaires were not validated for this population. To minimize this imprecision, we used food scores instead of assessing food intake at the food group level.

\section{Future Research}

While the obesogenic environment persists, consumers face a plethora of barriers to achieving a healthy body weight: food marketing, convenience of unhealthy choices, peer pressure, taste preference for calorie-dense foods, and many more [1]. Creating optimized interventions with a strong focus on diet, exercise, and factors that influence these can contribute to alleviating this problem [1]. These factors are easy to understand, and as first-line options for improving health, they are non-controversial $[18,65]$. It can be the role of researchers not just to formulate what optimal lifestyle choices would look like but also to optimize interventions which can effectively translate evidence-based knowledge into real-life health improvement [46].

While a healthy, more plant-based diet may have several health benefits [11], losing weight [66] and shifting away from a diet rich in animal-source foods both may have detrimental effects on bone health (particularly hip fracture risk) [67], especially if this dietary shift is associated with a decreased intake of key nutrients for bone 
health (including calcium, vitamin $\mathrm{D}$, and protein) [68], which may be the case in near-vegan diets [67]. Therefore, adequate sources of these key nutrients should be communicated to participants, if a predominantly plantbased diet is recommended [68].

\section{Conclusion}

Our intervention succeeded in achieving relatively long-term (1-year) improvements in body weight, BMI, WC, REM-C, and RHR. It failed at maintaining meaningful long-term improvements in any of the other parameters assessed. Participants were recruited from the general population in rural northwest Germany, and the findings are likely applicable to similar populations. The results indicate that our intervention is effective in terms of body weight but that the programme requires further optimization to effect long-term improvements in other risk markers.

\section{Acknowledgment}

We would like to thank all participants, Alwine Kraatz who took part in the investigation, and Dr. Norman Bitterlich for his help with statistical analysis.

\section{Statement of Ethics}

All subjects provided written informed consent before participating in the study. The study was conducted in accordance with the Declaration of Helsinki. The study protocol was approved by the Ethics Committee of the Medical Association of WestphaliaLippe and of the University of Münster (Münster, Germany; reference: 2018-171-f-S; approved on 4 April 2018).

\section{Conflict of Interest Statement}

The authors have no conflicts of interest to declare.

\section{Funding Sources}

This work was conducted as part of the project "münster.land. leben" and was funded by the German Federal Ministry of Education and Research (BMBF) and the Joint Science Conference (GWK) within the programme "Innovative Hochschule" (grant no.: 03IHS062A). The funders had no role in the study design, data collection, analysis, interpretation, writing of the report, or the decision to publish the findings. The publication of this article was funded by the Open Access Fund of Leibniz Universität Hannover.

\section{Author Contributions}

Christian Koeder: conceptualization, methodology, validation, formal analysis, investigation, data curation, writing - original draft, writing - review and editing, visualization, and project administration; Ragna-Marie Kranz: conceptualization, methodology, validation, investigation, data curation, writing - review and editing, and project administration; Corinna Anand: conceptualization, methodology, validation, investigation, data curation, writing - review and editing, and project administration; Sarah Husain: conceptualization, methodology, validation, investigation, data curation, writing - review and editing, and project administration; Dima Alzughayyar: investigation, data curation, and writing - review and editing; Nora Schoch: conceptualization, methodology, investigation, data curation, writing - review and editing, and project administration; Andreas Hahn: methodology, formal analysis, writing - original draft, writing - review and editing, and supervision; Heike Englert: conceptualization, methodology, investigation, writing - original draft, writing - review and editing, project administration, supervision, and funding acquisition.

\section{Data Availability Statement}

The data are available from the corresponding author (C.K.) upon reasonable request.

\section{References}

1 Blüher M. Obesity: global epidemiology and pathogenesis. Nat Rev Endocrinol. 2019;15: $288-98$.

2 Chooi YC, Ding C, Magkos F. The epidemiology of obesity. Metabolism. 2019;92:6-10.

3 Opio J, Croker E, Odongo GS, Attia J, Wynne K, McEvoy M. Metabolically healthy overweight/obesity are associated with increased risk of cardiovascular disease in adults, even in the absence of metabolic risk factors: a systematic review and meta-analysis of prospective cohort studies. Obes Rev. 2020;21:e13127.
4 Dwivedi AK, Dubey P, Cistola DP, Reddy SY. Association between obesity and cardiovascular outcomes: updated evidence from $\mathrm{Me}$ ta-analysis Studies. Curr Cardiol Rep. 2020; 22:25.

5 Chatterjee A, Gerdes MW, Martinez SG. Identification of risk factors associated with obesity and overweight: a machine learning overview. Sensors. 2020;20.
6 Morris MA, Wilkins E, Timmins KA, Bryant M, Birkin M, Griffiths C. Can big data solve a big problem? Reporting the obesity data landscape in line with the Foresight obesity system map. Int J Obes. 2018;42:1963-76.

7 Mehta NK, Strickling J, Mark E, Swinehart S, Puthumana J, Lavie CJ, et al. Beyond cardioversion, ablation and pharmacotherapies: risk factors, lifestyle change and behavioral counseling strategies in the prevention and treatment of atrial fibrillation. Prog Cardiovasc Dis. 2021 May-Jun;66:2-9.
Healthy Lifestyle Intervention to Address Overweight and Other Risk Markers 
8 Matsumoto S, Beeson WL, Shavlik DJ, Siapco G, Jaceldo-Siegl K, Fraser G, et al. Association between vegetarian diets and cardiovascular risk factors in non-hispanic white participants of the Adventist Health Study-2. J Nutr Sci. 2019;8:e6.

9 Burgess E, Hassmén P, Pumpa KL. Determinants of adherence to lifestyle intervention in adults with obesity: a systematic review. Clin Obes. 2017;7:123-35.

10 Abbate M, Gallardo-Alfaro L, Del Bibiloni MM, Tur JA. Efficacy of dietary intervention or in combination with exercise on primary prevention of cardiovascular disease: a systematic review. Nutr Metab Cardiovasc Dis. 2020;30:1080-93.

11 Cena H, Calder PC. Defining a healthy diet: evidence for the role of contemporary dietary patterns in health and disease. Nutrients. 2020;12(2):334

12 Baden MY, Liu G, Satija A, Li Y, Sun Q, Fung TT, et al. Changes in plant-based diet quality and total and cause-specific mortality. Circulation. 2019;140:979-91.

13 Betageri O, Al-Turk B, Freeman AM, Aggarwal M. Prevention and reversal of morbidity in today's cardiovascular patient: role of lifestyle modification and nutrition in the current era. Curr Cardiol Rep. 2021;23:143.

14 Mazzocchi A, Leone L, Agostoni C, PaliSchöll I. The secrets of the mediterranean diet. Does only olive oil matter? Nutrients. 2019;11(12):2941.

15 Najjar RS, Feresin RG. Plant-based diets in the reduction of body fat: physiological effects and biochemical insights. Nutrients. 2019;11.

16 Chopra S, Malhotra A, Ranjan P, Vikram NK, Singh N. Lifestyle-related advice in the management of obesity: a step-wise approach. J Educ Health Promot. 2020;9:239.

17 Wadden TA, Tronieri JS, Butryn ML. Lifestyle modification approaches for the treatment of obesity in adults. Am Psychol. 2020; 75:235-51.

18 Wilson K. Obesity: lifestyle modification and behavior interventions. FP Essent. 2020;492: 19-24.

19 Malin SK, Heiston EM, Gilbertson NM, Eichner NZM. Short-term interval exercise suppresses acylated ghrelin and hunger during caloric restriction in women with obesity. Physiol Behav. 2020;223:112978.

20 Melby CL, Paris HL, Foright RM, Peth J. Attenuating the biologic drive for weight regain following weight loss: must what goes down always go back up? Nutrients. 2017;9(4):68.

21 Fischer NM, Pallazola VA, Xun H, CainzosAchirica M, Michos ED. The evolution of the heart-healthy diet for vascular health: a walk through time. Vasc Med. 2020;25:184-93.

22 Tran E, Dale HF, Jensen C, Lied GA. Effects of plant-based diets on weight status: a systematic review. Diabetes Metab Syndr Obes. 2020;13:3433-48.

23 Stanulewicz N, Knox E, Narayanasamy M, Shivji N, Khunti K, Blake H. Effectiveness of lifestyle health promotion interventions for nurses: a systematic review. Int J Environ Res Public Health. 2020;17(1):17.

24 Lim S, Lee WK, Tan A, Chen M, Tay CT, Sood $S$, et al. Peer-supported lifestyle interventions on body weight, energy intake, and physical activity in adults: a systematic review and meta-analysis. Obes Rev. 2021;22(12):e13328.

25 Peiris CL, van Namen M, O’Donoghue G. Education-based, lifestyle intervention programs with unsupervised exercise improve outcomes in adults with metabolic syndrome. A systematic review and meta-analysis. Rev Endocr Metab Disord. 2021 Mar 17:1-14.

26 Odorico M, Le Goff D, Aerts N, Bastiaens H, Le Reste JY. How to support smoking cessation in primary care and the community: a systematic review of interventions for the prevention of cardiovascular diseases. Vasc Health Risk Manag. 2019;15:485-502.

27 Murtagh EM, Murphy MH, Milton K, Roberts NW, O'Gorman CS, Foster C. Interventions outside the workplace for reducing sedentary behaviour in adults under 60 years of age. Cochrane Database Syst Rev. 2020;7: CD012554.

28 Peachey MM, Richardson J, Tang VA, DalBello Haas V, Gravesande J. Environmental, behavioural and multicomponent interventions to reduce adults' sitting time: a systematic review and meta-analysis. Br J Sports Med. 2020;54:315-25.

29 Tam HL, Wong EML, Cheung K. Effectiveness of educational interventions on adherence to lifestyle modifications among hypertensive patients: an integrative review. Int J Environ Res Public Health. 2020;17(7):2513.

30 García-Molina L, Lewis-Mikhael AM, Riquelme-Gallego B, Cano-Ibáñez N, Oliveras-López MJ, Bueno-Cavanillas A. Improving type 2 diabetes mellitus glycaemic control through lifestyle modification implementing diet intervention: a systematic review and meta-analysis. Eur J Nutr. 2020;59: 1313-28.

31 Perez-Cueto FJA. An umbrella review of systematic reviews on food choice and nutrition published between 2017 and-2019. Nutrients. 2019;11:11.

32 Whatnall MC, Patterson AJ, Ashton LM, Hutchesson MJ. Effectiveness of brief nutrition interventions on dietary behaviours in adults: a systematic review. Appetite. 2018; 120:335-47.

33 Nagai M. Relationships among lifestyle awareness, age, and lifestyle-related diseases in healthy Japanese community residents. Asian Pac Isl Nurs J. 2020;5:103-10.

34 Ledderer L, Kjær M, Madsen EK, Busch J, Fage-Butler A. Nudging in public health lifestyle interventions: a systematic literature review and metasynthesis. Health Educ Behav. 2020;47:749-64.

35 Collado-Mateo D, Lavín-Pérez AM, Peñacoba C, Del Coso J, Leyton-Román M, LuqueCasado A, et al. Key factors associated with adherence to physical exercise in patients with chronic diseases and older adults: an umbrella review. Int J Environ Res Public Health. 2021;18(4):2023.

36 Nightingale BA, Cassin SE. Disordered eating among individuals with excess weight: a review of recent research. Curr Obes Rep. 2019; 8:112-27.

37 Pearl RL, Puhl RM, Himmelstein MS, Pinto AM, Foster GD. Weight stigma and weightrelated health: associations of self-report measures among adults in weight management. Ann Behav Med. 2020;54:904-14.

38 van Namen M, Prendergast L, Peiris C. Supervised lifestyle intervention for people with metabolic syndrome improves outcomes and reduces individual risk factors of metabolic syndrome: a systematic review and metaanalysis. Metabolism. 2019;101:153988.

39 Shirinzadeh M, Afshin-Pour B, Angeles R, Gaber J, Agarwal G. The effect of communitybased programs on diabetes prevention in low- and middle-income countries: a systematic review and meta-analysis. Global Health. 2019;15:10.

40 Koeder C, Hahn A, Englert H. Effect of a 6-month controlled lifestyle intervention on common carotid intima-media thickness. J Nutr Health Aging. 2021;25(7):869-77.

41 Parker K, Parikh SV. Applying Prochaska's model of change to needs assessment, programme planning and outcome measurement. J Eval Clin Pract. 2001;7:365-71.

42 Nakabayashi J, Melo GR, Toral N. Transtheoretical model-based nutritional interventions in adolescents: a systematic review. BMC Public Health. 2020;20:1543.

43 Prochaska JO, Velicer WF. The transtheoretical model of health behavior change. Am J Health Promot. 1997;12:38-48.

44 Yoshida-Montezuma Y, Ahmed M, Ezezika $\mathrm{O}$. Does gamification improve fruit and vegetable intake in adolescents? A systematic review. Nutr Health. 2020;26:347-66.

45 Dos Santos Q, Perez-Cueto FJA, Rodrigues VM Appleton K, Giboreau A, Saulais L, et al. Impact of a nudging intervention and factors associated with vegetable dish choice among European adolescents. Eur J Nutr. 2020;59:231-47.

46 Satija A, Bhupathiraju SN, Spiegelman D, Chiuve SE, Manson JE, Willett W, et al. Healthful and unhealthful plant-based diets and the risk of coronary heart disease in US adults. J Am Coll Cardiol. 2017;70:411-22.

47 Curry SI, Curry SI, Krist AH, Owens DK, Barry $\mathrm{MJ}$, Caughey $\mathrm{AB}$, et al. Behavioral weight loss interventions to prevent obesity-related morbidity and mortality in adults: US preventive services task force recommendation statement. JAMA. 2018;320:1163-71.

48 Durrer Schutz D, Busetto L, Dicker D, Farpour-Lambert N, Pryke R, Toplak H, et al. European practical and patient-centred guidelines for adult obesity management in primary care. Obes Facts. 2019;12:40-66.

49 Verboven K, Hansen D. Critical reappraisal of the role and importance of exercise intervention in the treatment of obesity in adults. Sports Med. 2021;51(3):379-89. 
50 Oussalah A, Levy J, Berthezène C, Alpers DH, Guéant JL. Health outcomes associated with vegetarian diets: an umbrella review of systematic reviews and meta-analyses. Clin Nutr. 2020;39:3283-307.

51 Zinöcker MK, Svendsen K, Dankel SN. The homeoviscous adaptation to dietary lipids (HADL) model explains controversies over saturated fat, cholesterol, and cardiovascular disease risk. Am J Clin Nutr. 2021;113(2): 277-89.

52 Sakamoto M, Matsutani D, Minato S, Tsujimoto Y, Kayama Y, Takeda N, et al. Seasonal variations in the achievement of guideline targets for $\mathrm{HbA1c}$, blood pressure, and cholesterol among patients with type 2 diabetes: a Nationwide Population-Based Study (ABC Study: JDDM49). Diabetes Care. 2019;42: 816-23.

53 Kumar BV, Mohan T. Sigma metrics as a tool for evaluating the performance of internal quality control in a clinical chemistry laboratory. J Lab Physicians. 2018;10:194-9.

54 Floegel A, Kühn T, Sookthai D, Johnson T, Prehn C, Rolle-Kampczyk U, et al. Serum metabolites and risk of myocardial infarction and ischemic stroke: a targeted metabolomic approach in two German prospective cohorts. Eur J Epidemiol. 2018;33:55-66.

55 Katzke VA, Sookthai D, Johnson T, Kühn T, Kaaks R. Blood lipids and lipoproteins in relation to incidence and mortality risks for CVD and cancer in the prospective EPIC-heidelberg cohort. BMC Med. 2017;15:218.
56 Bonfiglio C, Leone CM, Silveira LVA, Guerra R, Misciagna G, Caruso MG, et al. Remnant cholesterol as a risk factor for cardiovascular, cancer or other causes mortality: a competing risks analysis. Nutr Metab Cardiovasc Dis. 2020;30:2093-102.

57 Castañer O, Pintó X, Subirana I, Amor AJ, Ros E, Hernáez Á, et al. Remnant cholesterol, not LDL cholesterol, is associated with incident cardiovascular disease. J Am Coll Cardiol. 2020;76:2712-24.

58 Jepsen AM, Langsted A, Varbo A, Bang LE, Kamstrup PR, Nordestgaard BG. Increased remnant cholesterol explains part of residual risk of all-cause mortality in 5414 patients with ischemic heart disease. Clin Chem. 2016; 62:593-604.

59 Salinas CAA, Chapman MJ. Remnant lipoproteins: are they equal to or more atherogenic than LDL? Curr Opin Lipidol. 2020;31: 132-9.

60 Campanella A, Iacovazzi PA, Misciagna G, Bonfiglio C, Mirizzi A, Franco I, et al. The effect of three mediterranean diets on remnant cholesterol and non-alcoholic fatty liver disease: a secondary analysis. Nutrients. 2020;12.

61 Seravalle G, Grassi G. Heart rate as cardiovascular risk factor. Postgrad Med. 2020;132: 358-67.
62 Aune D, Sen A, Ó'Hartaigh B, Janszky I, Romundstad PR, Tonstad S, et al. Resting heart rate and the risk of cardiovascular disease, total cancer, and all-cause mortality: a systematic review and dose-response meta-analysis of prospective studies. Nutr Metab Cardiovasc Dis. 2017;27:504-17.

63 Huang J, Liao J, Fang Y, Deng H, Yin H, Shen $B$, et al. Six-week exercise training with dietary restriction improves central hemodynamics associated with altered gut microbiota in adolescents with obesity. Front Endocrinol. 2020;11:569085.

64 Reddy YNV, Anantha-Narayanan M, Obokata M, Koepp KE, Erwin P, Carter RE, et al. Hemodynamic effects of weight loss in obesity: a systematic review and meta-analysis. JACC Heart Fail. 2019;7:678-87.

65 Khandelwal S. Obesity in midlife: lifestyle and dietary strategies. Climacteric. 2020;23:1407.

66 Wing RR. Does lifestyle intervention improve health of adults with overweight/obesity and type 2 diabetes? Findings from the look AHEAD randomized trial. Obesity. 2021; 29(8):1246-58

67 Tong TYN, Appleby PN, Armstrong MEG, Fensom GK, Knuppel A, Papier K, et al. Vegetarian and vegan diets and risks of total and site-specific fractures: results from the Prospective EPIC-Oxford Study. BMC Med. 2020;18:353

68 Aggarwal R, Bains K. Protein, lysine and vitamin D: critical role in muscle and bone health. Crit Rev Food Sci Nutr. 2020;1-12:1. 